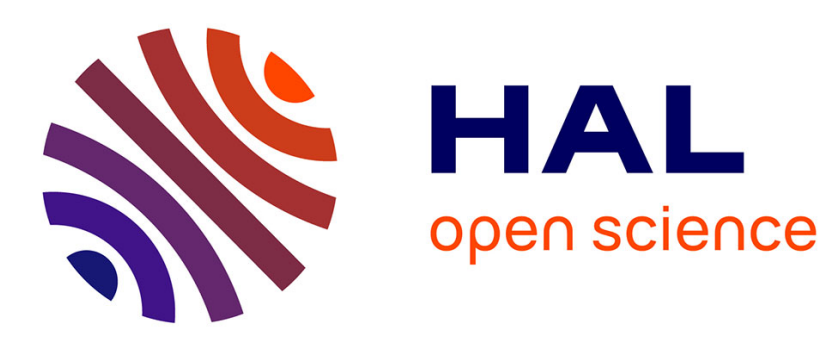

\title{
Influence of imperfect joints and geometrical tolerances on a circuit breaker dynamics
}

\author{
Narendra Akhadkar, Vincent Acary, Bernard Brogliato
}

\section{To cite this version:}

Narendra Akhadkar, Vincent Acary, Bernard Brogliato. Influence of imperfect joints and geometrical tolerances on a circuit breaker dynamics. IFToMM WC 2019: Advances in Mechanism and Machine Science, Jun 2019, Krakow, Poland. pp.3069-3078, 10.1007/978-3-030-20131-9_302 . hal-02440880

\section{HAL Id: hal-02440880 \\ https://hal.inria.fr/hal-02440880}

Submitted on 11 Jun 2020

HAL is a multi-disciplinary open access archive for the deposit and dissemination of scientific research documents, whether they are published or not. The documents may come from teaching and research institutions in France or abroad, or from public or private research centers.
L'archive ouverte pluridisciplinaire $\mathbf{H A L}$, est destinée au dépôt et à la diffusion de documents scientifiques de niveau recherche, publiés ou non, émanant des établissements d'enseignement et de recherche français ou étrangers, des laboratoires publics ou privés. 


\title{
Influence of imperfect joints and geometrical tolerances on a circuit breaker dynamics
}

\author{
Narendra Akhadkar ${ }^{1}$, Vincent Acary ${ }^{2}$, and Bernard Brogliato ${ }^{2}$ \\ ${ }^{1}$ Schneider Electric, 2 Chemin des sources, 38240, Meylan, France, \\ narendra.akhadkarese.com \\ ${ }^{2}$ INRIA Grenoble Rhône-Alpes and Laboratoire Jean Kuntzman, University Grenoble-Alpes, \\ 655 avenue de l'Europe, 38334 Saint-Ismier, France, vincent . acaryeinria.fr, \\ bernard.brogliato@inria.fr
}

\begin{abstract}
The aim of this paper is to understand the influence of clearance in the kinematic joints, dimensional and geometrical tolerances associated with the parts on the performance of a circuit breaker mechanism in the trip operation. Operating mechanism and trip unit are the essential components of a miniature circuit breaker. The operating and trip mechanism are made of ten parts with revolute and cylindrical joints with clearance and five unilateral contacts with friction. This mechanism is based on quick-make and quick-break principle. The MoreauJean nonsmooth contact dynamics (NSCD) numerical method is used to perform the simulations. The numerical results are validated by careful comparisons with experimental data.
\end{abstract}

Keywords: Joint clearance; geometrical tolerances; unilateral constraints; Coulomb's friction; impacts; circuit breaker.

\section{Introduction}

The important function of circuit breaker is to switch ON/OFF/TRIP the electrical current and to protect the lowest common distributed voltage in an electrical system. It plays a vital role to safegaurd the electrical system in the event of electrical short circuit and overload conditions. Trip unit activates the electro-magnetic tripping mechanism to break the current flow in the electrical system/network. The time duration to break the current flow is few milliseconds and any delay in tripping function can create hazardous conditions where human safety can not be guaranteed. Usually, the performance of circuit breaker mechanism is not as desired, due to various factors such as manufacturing dimensional and geometrical tolerances on the parts, clearances in the joints and the assembly tolerances. These factors are directly linked to the manufacturing cost of the product and it is important to optimize the product cost and to gurantee the desired overall performance of the product. In the computer models it is always assumed that the geometry of the real part is perfect. In reality the real surfaces are subject to irregularities such as bumps, undulations and surface roughness [1]. Geometric tolerances are always defined by the tolerance band with upper and lower limit of acceptance. It is assumed that the real surface must lie inside this tolerance band $[1,8,12]$. 
Clearance in the spatial revolute (cylindrical) joint adds five (four) extra degrees of freedom into the system. Most of the previous work is focused on the radial clearance in the planar and spatial revolute and cylindrical joints $[10,14,13]$. However more recently the influence of the axial clearance in the revolute joint has been studied in $[18,19]$.

Most of the mechanisms in the Schneider Electric company use frictional contacts and the compliant models cannot correctly model the sticking condition. In the nonsmooth contact dynamic (NSCD) approach, the interaction of the colliding bodies is modeled with multiple frictional unilateral constraints $[15,16]$. The unilateral constraints are described by set-valued force laws in normal and tangential directions. The normal contact law is based on Signorini's condition while the tangential contact law is based on Coulomb's friction law. Careful comparisons between numerical results obtained with the NSCD approach, and experimental data are reported in $[17,20]$, while the use of the NSCD approach for systems with clearances is also advocated in $[11,6]$. They demonstrate that the numerical schemes and the model used in this article, though they can be improved, possess very good forecast capabilities.

Our aim is to study the influence of clearance in the kinematic joints, dimensional and geometrical tolerances on the tripping function of the miniature circuit breaker in the three dimensional case. Another objective is to develop a virtual test bench using the INRIA open-source simulation software SICONOS ${ }^{1}$. Our aim is to validate the simulation model, experiments are carried-out on the prototype samples and the results are compared with the simulations.

\section{Formulation of the nonsmooth dynamical systems}

\subsection{Normal and tangential contact laws}

Let us consider two non overlapping bodies (see Fig. 1), a potential contact point between two bodies is given by the closest points $C_{A}$ and $C_{B}$. A local frame is defined at the potential contact point by $\left(\mathrm{N}, \mathrm{T}_{1}, \mathrm{~T}_{2}\right)$. The gap $g_{\mathrm{N}}$ is defined a the signed distance between the two potential contacting points $C_{A}$ and $C_{B}$. The con-

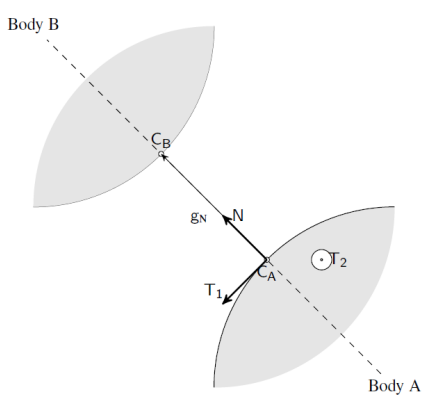

Fig. 1: Contact local frame.

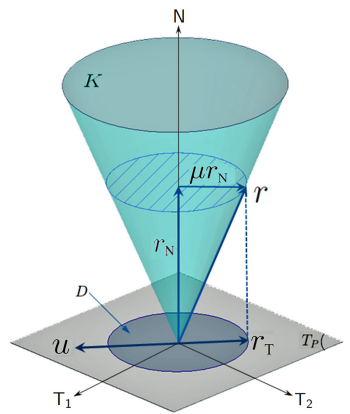

Fig. 2: 3D Coulomb's friction cone, sliding case.

\footnotetext{
${ }^{1} \mathrm{http} / / /$ siconos.gforge.inria.fr/
} 
tact force, denoted by $r=\left(r_{\mathrm{N}}, r_{\mathrm{T}}\right)^{\top} \in \mathbb{R}^{3}$. Due to the impenetrability assumption one has $g_{\mathrm{N}} \geqslant 0$. We also neglect adhesive effects so that $r_{\mathrm{N}} \geqslant 0$. If $r_{\mathrm{N}}>0$ then we impose $g_{\mathrm{N}}=0$, and when $g_{\mathrm{N}}>0$, the normal contact force must vanish, i.e. $r_{\mathrm{N}}=0$ (no magnetic or distance forces) $[2,4,7]$. These conditions yield a complementarity condition denoted compactly as:

$$
0 \leqslant g_{\mathrm{N}} \perp r_{\mathrm{N}} \geqslant 0 .
$$

The normal contact law at the velocity level is expressed as :

$$
0 \leqslant u_{\mathrm{N}} \perp r_{\mathrm{N}} \geqslant 0, \quad \text { if } g_{\mathrm{N}}=0 .
$$

The tangential contact law is the Coulomb friction that constrain the contact force $r$ in the the friction cone (see Fig. 2)

$$
r \in K=\left\{r \in \mathbb{R}^{3},\left\|r_{\mathrm{T}}\right\| \leqslant \mu r_{\mathrm{N}}\right\} .
$$

The scalar $\mu \geqslant 0$ is the coefficient of friction. In case of sliding the tangential force $r_{\mathrm{T}}$ acts in direction opposite to the relative tangential velocity $u_{\mathrm{T}}$. If the relative tangential velocity $u_{\mathrm{T}}$ is zero then the bodies stick to each other (rolling without slipping). We introduce the modified relative velocity $\widehat{u}:=u+\mu\left\|u_{\mathrm{T}}\right\| \mathrm{N}$, then the Coulomb friction can be equivalently expressed as a second-order cone complementarity condition $[9,5]$ if $g_{\mathrm{N}}=0$ :

$$
K^{*} \ni \widehat{u} \perp r \in K .
$$

The cone $K^{*}=\left\{v \in \mathbb{R}^{3} \mid r^{T} v \geqslant 0, \forall r \in K\right\}$ is the dual cone of $K$.

\subsection{Newton-Euler formulation of the equation of motion}

Let us consider a mechanical system subjected to $m$ constraints, with $m_{e}$ holonomic bilateral constraints $h^{\alpha}(q)=0, \alpha \in \mathscr{E} \subset \mathbb{N}, m_{i}$ unilateral constraints $g_{\mathrm{N}}^{\alpha}(q) \geqslant$ $0, \alpha \in \mathscr{I} \subset \mathbb{N}$ and Coulomb friction. The Newton-Euler formulation of such a system is given as:

$$
\left\{\begin{array}{ll}
\dot{q}=T(q) v, & \\
M \dot{v}=F(t, q, v)+H^{\top}(q) \lambda+G^{\top}(q) r, & \alpha \in \mathscr{E} \\
H^{\alpha}(q) v=0, & \widehat{u}^{\alpha}=u^{\alpha}+\mu^{\alpha}\left\|u_{\mathrm{T}}^{\alpha}\right\| \mathrm{N}^{\alpha} \\
u^{\alpha}=G^{\alpha}(q) v, & \text { if } g_{\mathrm{N}}^{\alpha}(q)>0, \\
r^{\alpha}=0, & \text { if } g_{\mathrm{N}}^{\alpha}(q)=0, \\
K^{\alpha, *} \ni \widehat{u}^{\alpha} \perp r^{\alpha} \in K^{\alpha} & \\
u_{\mathrm{N}}^{\alpha,+}=-e_{r}^{\alpha} u_{\mathrm{N}}^{\alpha,-}, & \text { if } g_{\mathrm{N}}^{\alpha}(q)=0 \text { and } u_{\mathrm{N}}^{\alpha,-} \leqslant 0,
\end{array}\right\} \alpha \in \mathscr{I}
$$

where $q$ is the vector of coordinates of the position and the orientation of the body, $v$ is the velocity, the operator $T(q) \in \mathbb{R}^{7 \times 6}$ links the time derivatives of the coordinates to the velocities, $M$ is the total inertia matrix, $F(t, q, v) \in \mathbb{R}^{6}$ collects all the forces and torques applied to the body. The operators $H \in \mathbb{R}^{m_{e} \times n}$ and $G \in \mathbb{R}^{3 m_{i} \times n}$ link the local velocity variables in the joints, and at contacts respectively, to the velocity vector of the bodies. 


\subsection{The numerical integration method}

In this paper we use the event-capturing method based on the Moreau-Jean time-stepping scheme $[4,15,16]$, where the constraints are solved at the velocity level and thereafter named the NSCD method. It is well-known that velocity level treatment of constraints yields violations of constraints with the drift phenomenon. When we simulate mechanisms with small clearances, this is not tolerable since we have to keep the violation as small as possible with respect to the characteristic length of the clearances. To overcome this limitation of the standard Moreau-Jean time-stepping scheme, we use the combined projection scheme as proposed in [3].

\section{The C-60 miniature circuit breaker mechanism}

Miniature circuit breaker consist of operating mechanism, trip unit, arc chute, electrical contacts enclosed in insulated housing. Important functions of circuit breaker are: to SENSE the overcurrent in the electrical network, MEASURE the amount of overcurrent flowing and to ACT by tripping the operating mechanism to break the contacts in a timely manner to ensure human safety and to prevent damage. A typical miniature circuit breaker mechanism with trip unit is depicted

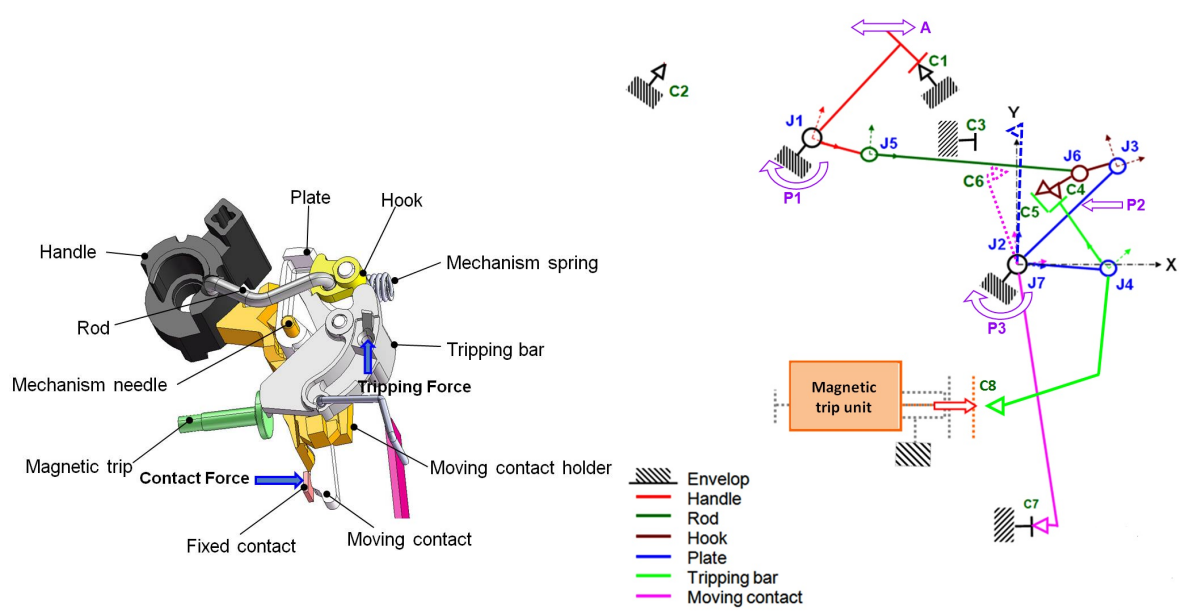

(a) $3 \mathrm{D}$ view.

(b) Kinematic representation.

Fig. 3: C-60 circuit breaker mechanism - ON position.

in Fig. 3(a).

Mechanism working principle: All the mechanism parts are enclosed in-between the case and cover parts. These parts are connected to each other through a revolute joint or frictional contact. In the following section we will see the detailed description of these kinematic joints and contacts. In the first step, the primary 
function of a mechanism is usually formulated in terms of kinematical quantities (link geometry, kinematic constraints, etc). Also the various geometrical relations resulting from the kinematical analysis of the linkage mechanism are an essential ingredient for the dynamic analysis. The kinematical analysis of a miniature circuit breaker mechanism (hereafter called the C-60 mechanism) is of great importance. The C-60 operating mechanism consists of seven links, seven revolute joints with clearance in both radial and axial direction and four frictional contacts (see Fig. 3(b)). It has 42 degrees of freedom. The trip unit through trip mechanism determines when the contacts will open automatically. A trip mechanism is held in place by the tripper bar (see Fig. 3(a)). As long as the tripper bar holds the trip mechanism, the mechanism remains firmly locked in place and remains in $\mathrm{ON}$ position.

The operating mechanism in the ON position is explained as follows:

The close operation leads to ON position of the breaker. In close operation (see Fig. 3(b)), the operating handle $(A)$ is rotated clockwise which closes the contacts $C_{5}$ and $C_{4}$ through the revolute joints $J_{1}, J_{5}, J_{6}, J_{3}$ and $J_{4}$. The frictional contacts $C_{5}$ and $C_{4}$ have a specific wedge shape profile, which enables the locking between the hook and tripping bar. After the activation of the contacts $C_{5}$ and $C_{4}$ the motion has been transferred to the moving contact through the plate by revolute joints $J_{2}$ and $J_{7}$, which ensure closing of the contact between the moving and the fixed contact. During close operation the handle spring $\left(P_{1}\right)$ and the mechanism springs $\left(P_{2}\right.$ and $\left.P_{3}\right)$ get charged, which will be used for the trip operation of the breaker.

The Trip operation leads to TRIP position of the breaker. In the trip operation (see Figure 3(b)), the activation of the tripping coil causes the tripping plunger to be attracted, thus releasing the contact $C_{7}$ through $C_{3}$ and $C_{4}$. In response, the handle and mechanism spring discharge by moving the handle and plate to the open position.

\subsection{Revolute joint between the case, the cover and the handle: $J_{1}$}

The revolute joint between the case, the cover and the handle is $J_{1}$. The protrusion on the case and cover acts as a journal and the cavity on the handle acts as a bearing. In our modelling approach the ideal revolute joint is replaced by a journal and two circular rings at the extreme ends of the bearing (see Fig. 4) which acts as a spatial revolute joint with clearance. The axial and radial clearances in the revo-

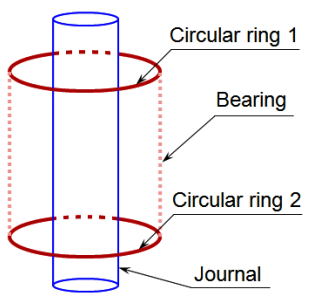

Fig. 4: Revolute joint with clearance $J_{1}$.

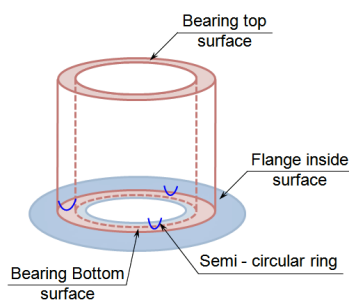

Fig. 5: Modeling of plane-plane contact. 
lute joint are modeled by introducing six degrees of freedom between the bearing and the journal. The relative motion between them is restricted by the internal surface of the bearing and the flanges of the journal. The radial clearance can be varied by changing the internal diameter of the bearing. The contact between the flange and the bearing top/bottom surface is a plane-plane contact. In reality, a plane-plane contact is impossible due to the presence of surface roughness and waviness. However the plane-plane contact can be completely described by three contact points. Few limitations of the plane-plane contacts are: more simulation time is required (numerically costly) as the contact detection is done on the entire area, and in return it gives only one contact point between the plane-plane contact which is practically not correct. The contact between the flange and the bearing face is modeled by considering the plane surface of the flange, while the plane surface of the bearing is replaced by three semi-circular equidistant rings (see Fig. 5).

\subsection{Geometrical tolerances}

The aim is to study the influence of the position and perpendicularity tolerances on the C-60 breakers performance (see Figures 6(a)-(b) and 7(a)-(b)). In case of the orientation tolerance of perpendicularity, the value of tolerance is defined by the zone of tolerance which is bounded by two parallel planes separated by a distance $t$, and perpendicular to the datum surface ${ }^{2}$ (see Figure 6(a)). In case of axis perpendicularity, the value of the tolerance is defined by the zone of tolerance which is bounded by a cylinder of diameter $t$, perpendicular to the datum plane, if the tolerance is preceded by the symbol $\phi$ (see Figure 6(b)). The position tolerance can be divided in two types such as, simple and bidirectional position. In case of the simple position tolerance, the zone of tolerance is bounded by two parallel planes at a distance $t$, and placed symmetrically with respect to the theoretically exact position of the surface under consideration (see Figure 7(a)). In the bidirectional position tolerance, the value of tolerance is defined by the zone of tolerance which is bounded by a cylinder of diameter $t$, whose axis is in the theoretically exact position of the line under consideration, if the value of tolerance is preceded by the symbol $\phi$ (see Figure 7(b)).

\subsection{Modeling of the geometrical tolerances in SICONOS}

The position tolerance is modeled by varying the position of the bearing axis from the reference axis (datum) (see Figure 8(a)). We have considered the two extreme cases, i.e., LSL and USL of the position tolerance on the axis of bearing. With these two axis positions we can create the 3D CAD model of the bearing. In Figure 8(a), the new locations of the bearing are shown with blue and red color dotted lines along with the new bearing axis. In this way we can simulate two different cases of the geometrical tolerances. Similarly for the perpendicularity tolerance, the journal axis can be anywhere between the tolerance band (see Figure 6(b)). For example, a particular case is depicted in Figure 8(b), where the journal axis is oriented at an angle from the reference plane. The CAD model is developed for this case and used for the simulations. The major conclusions are: (i) In case of

\footnotetext{
${ }^{2} \mathrm{~A}$ datum surface/plane is the theoretical exact plane/surface from which the vertical distances of the points above or below of this surface are measured.
} 


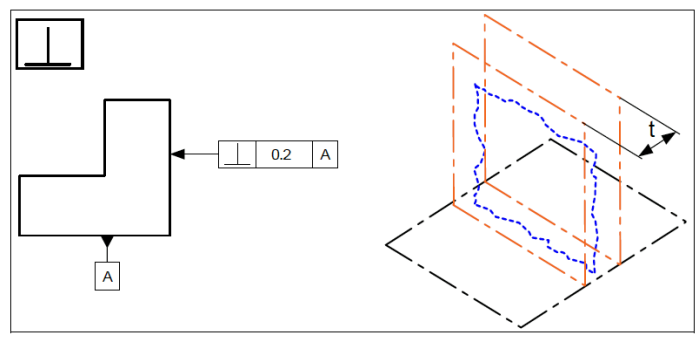

(a) Surface perpendicularity.

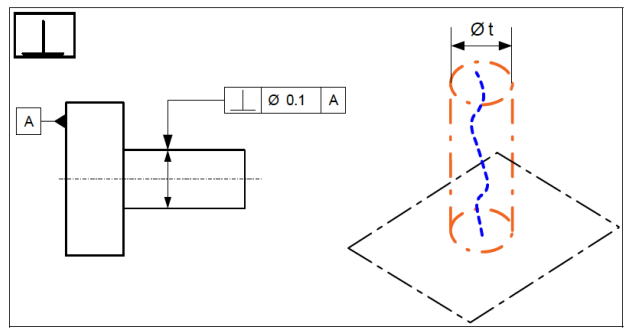

(b) Axis perpendicularity

Fig. 6: Orientation tolerance: Perpendicularity.

Table 1: Comparison between the influence of radial clearance and geometrical tolerance.

\begin{tabular}{|c|c|c|c|c|c|}
\hline \multirow{3}{*}{$\begin{array}{l}\text { Functional } \\
\text { condition }\end{array}$} & \multicolumn{3}{|c|}{$\begin{array}{l}\text { Equal radial clearance in all the } \\
\text { joints }(\mathrm{mm})\end{array}$} & \multicolumn{2}{|c|}{$\begin{array}{l}\% \text { relative error } \\
A^{\text {ref }}-A^{c / g}\end{array}$} \\
\hline & \multirow{2}{*}{$0.01 \mathrm{~mm}\left(A^{\text {ref }}\right)$} & \multirow{2}{*}{$0.05 \mathrm{~mm}\left(A^{c}\right)$} & \multirow{2}{*}{$\begin{array}{c}0.05 \mathrm{~mm}+\text { Geometrical } \\
\text { tolerance }\left(A^{g}\right)\end{array}$} & & \\
\hline & & & & $e_{c}$ & $e_{g}$ \\
\hline FC-3 & 7.526 & 6.935 & 6.581 & -7.85 & -15.56 \\
\hline FC-4 & 10.749 & 10.710 & 10.501 & -0.37 & -2.30 \\
\hline FC-5 & 4.534 & 4.269 & 4.102 & -5.84 & -9.54 \\
\hline FC-6 & 0.421 & 0.628 & 0.671 & 49.17 & 59.38 \\
\hline FC-7 & 10.587 & 10.459 & 10.351 & -1.21 & -2.22 \\
\hline FC-8 & 2.879 & 2.681 & 2.558 & -6.88 & -11.15 \\
\hline
\end{tabular}




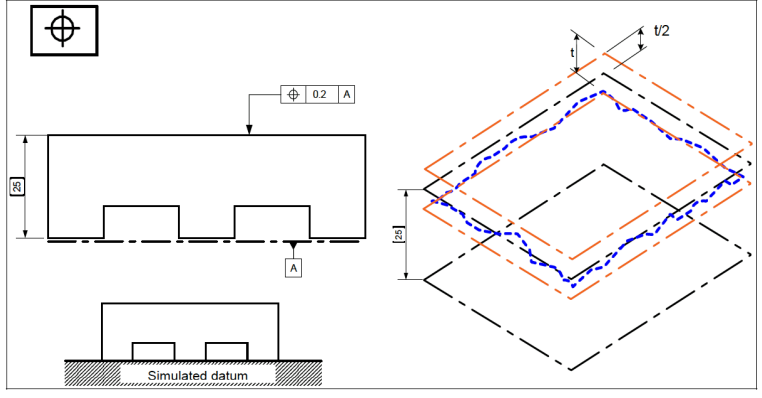

(a) Surface position.

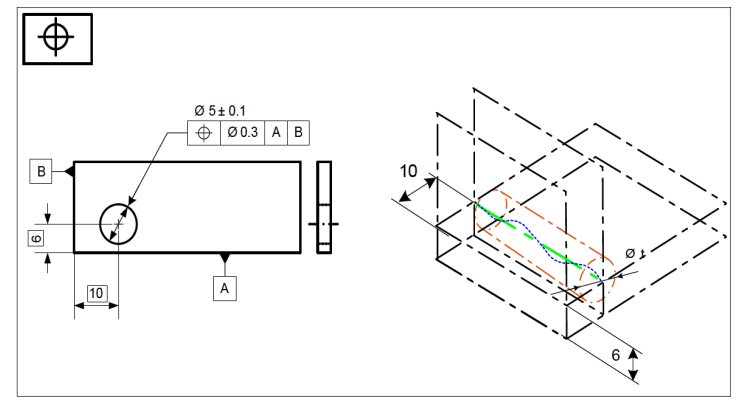

(b) Axis position.

Fig. 7: Position tolerance. 


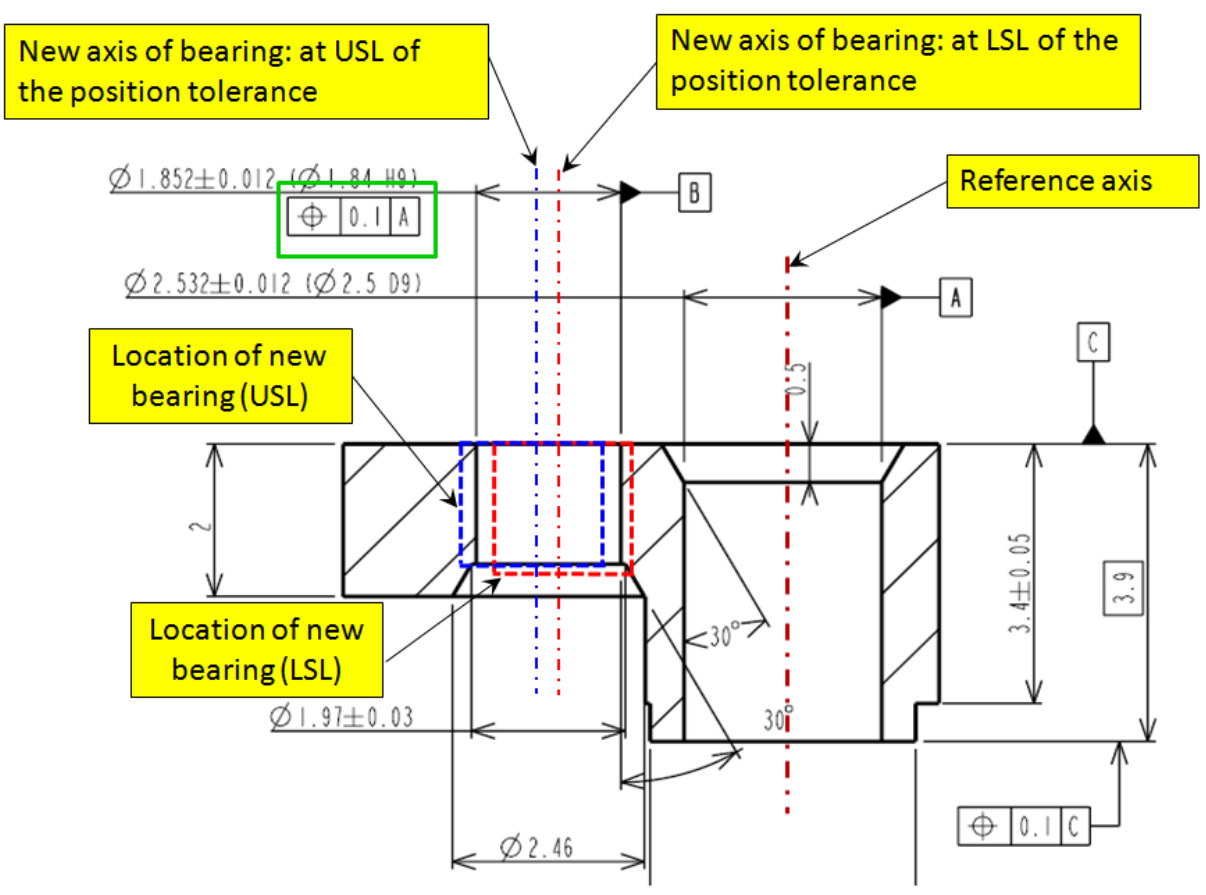

(a) Position tolerance.

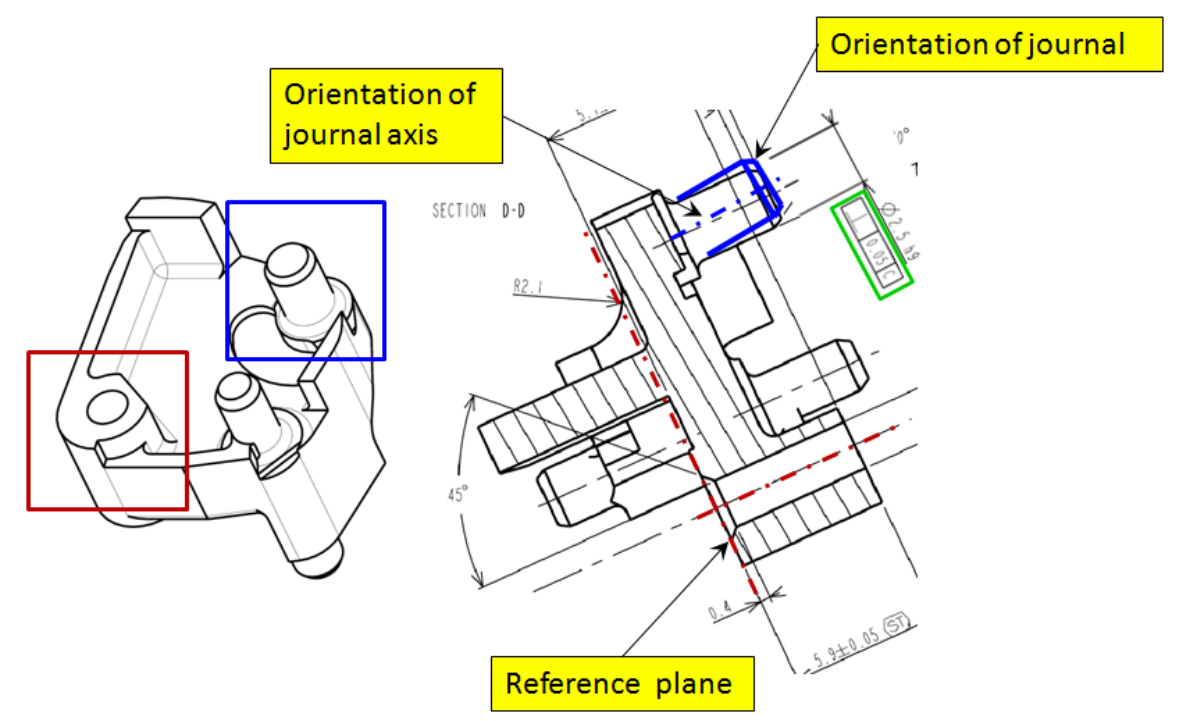

(b) Perpendicularity tolerance.

Fig. 8: Modeling of the geometrical tolerances in SICONOS. 
the dimensional and geometrical variation combined together, approximately the maximum variation of $10 \%$ is observed on the FC- 6 when compared to the case dimensional tolerance (see Table 1). The magnetic tripping distance increases as the radial clearance and the geometrical variation increase. (ii) In case of the FC-3, FC-4, FC-5, FC-7 and FC-8 the total variation less than 5\% is observed between the case of dimensional and dimensional with geometrical (combined) tolerance (see Table 1). (iii) The variations in the functional conditions due to geometrical tolerances may increase if all the geometrical variations (position, orientation and form tolerances) are considered together. However the process of modeling all these variations in the CAD model are quite tedious and challenging.

\section{Experimental validation: Contact force versus displacement}

In this section we report comparisons between numerical results obtained with the NSCD method, and experimental data obtained on physical prototypes built by Schneider Electric. The radial clearance in the revolute joints is given as: $J_{1}=$ $0.085 \mathrm{~mm}, J_{2}=0.05 \mathrm{~mm}, J_{3} / J_{4}=0.06 \mathrm{~mm}, J_{5} / J_{6}=0.045 \mathrm{~mm}$ and $J_{7}=0.055 \mathrm{~mm}$. Referring to the arrow in Fig. 3, the comparisons are made by recording force and displacement histories at the moving contact. The test bench consists of the

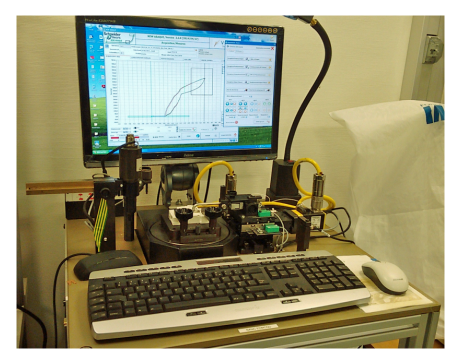

Fig. 9: Experimental test bench for contact/tripping force measurement.

fixture to mount the C-60 breaker and the moving table which comprises a pair of linear motion guide, see Fig. 9. The load cell is mounted on the moving table to measure the force and the bi-axial movement of the moving table is measured by two position sensors. The contact force of the moving contact $C_{7}$ (see Fig. 3(b)) is measured with the help of load cell, and is recorded by the computer programme. We have followed a similar methodology for the virtual testing (virtual test bench) of the C-60 product using the simulation.

1. In case of experimental test, the effect of polarization of the joints is approximately less by $50 \%$ when compared to the total displacement of the moving contact. At the static equilibrium (at the end of forward motion) of the sample-1, the recorded contact force is $15.28 \mathrm{~N}$ and the total displacement is $2.0 \mathrm{~mm}$, see Fig. 10(a). The results of the experimental test are compared with the numerical simulation. In case of the numerical test the effect of 


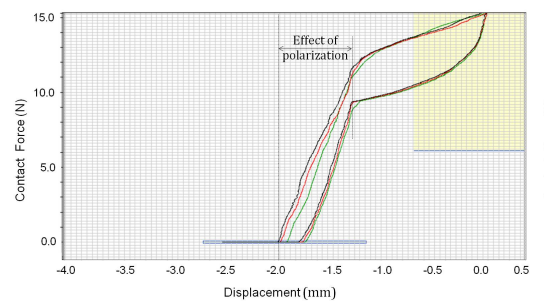

(a) Experimental result.

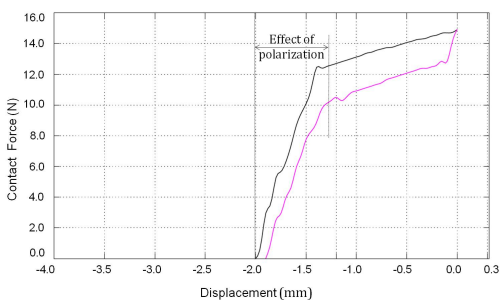

(b) Simulation result.

Fig. 10: Sample-1: Contact force versus Displacement.

polarization is similar to that of the experimental test, see Fig. 10(b). However the trajectory of the contact force in both the experiment and numerical tests are not identical, this may be due to the fact that the geometrical variations on the contacting surfaces of the parts are not considered in the case of numerical simulation. In reality the geometrical variations always exist and these variations may change the polarization of the parts (contact points between the parts). In case of the numerical simulation, the contact force at the static equilibrium is $14.96 \mathrm{~N}$. The percentage relative error in the contact force between the experiment and numerical test is $2.08 \%$.

2. The trajectory of the contact force in case of forward motion is lagging behind the backward motion of the moving contact. This is due to the effects of friction (change in the direction of the frictional forces) in the joints. In case of the numerical simulation, the coefficient of friction between the plasticplastic materials is considered to be $\mu=0.3$, and between the steel-plastic materials $\mu=0.1$ In case of experimental test the real values of the coefficient of friction are not known. This may be one of the reasons behind the slightly different behaviour of the contact force trajectories between the experiment and virtual test.

A rectangular shaped opening on the front and back surface of the tripping bar is present on the case and cover of the $\mathrm{C}-60$ product. The cavity on the tripping bar on the cover side is called as pin-side and the cavity on the case side is called as lamage-side. The tripping operation is possible only if the product is in ON condition.

In the case of pin-side tripping, the position of the cavity on the tripping bar is recorded by the plunger/probe and set as the reference position. When the plunger/probe comes in contact with the tripping bar cavity, a small load is detected by the load cell. This is the starting point of the test. Now with the help of computer program the linear motion of $(0.5 \mathrm{~mm} / \mathrm{s})$ is given to the plunger through the moving table and the constant velocity is ensured by the controller. The tripping bar is pushed forward (in positive $\mathrm{Y}$ direction) to break the frictional contact between the hook and the tripping bar $\left(C_{4}\right)$ and the corresponding distance and the tripping force is recorded by the position sensor and the load cell respectively (see Figure 9(b)). Similar methodology is followed for the lamage side tripping.

For the virtual test, we have performed some iterations to find out the approximate location of the potential contact points similar to the prototype model. In doing 


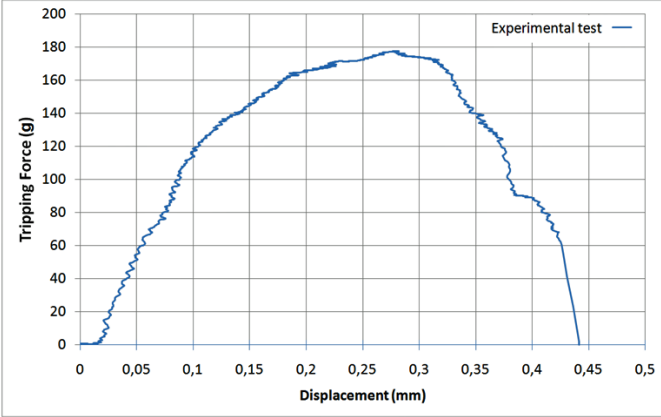

(a) Experimental test

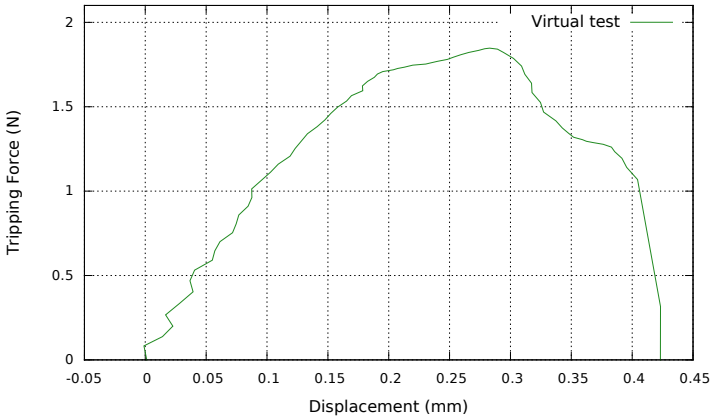

(b) Virtual test

Fig. 11: Tripping force vs Displacement: pin-side.

so, the experimental results helped us to predict the possible contacts in the hook and tripping bar joint. Also we have captured the magnified images of the contact surfaces to understand the profile of the surface. All this inputs are used to locate approximately the potential contacts in the hook and tripping bar contact. The major conclusions are: (i) In case of the experimental results, the recorded peak force is $1.77 \mathrm{~N}$ at a distance of $0.27 \mathrm{~mm}$. The total trip distance is $0.44 \mathrm{~mm}$ (see Figure 11(a)). In case of the virtual test, the trajectory of the tripping force is slightly different when compared to the experimental results (see Figure 11(b)). The total trip distance is $0.42 \mathrm{~mm}$, and the variation of $0.02 \mathrm{~mm}$ is seen between the virtual and experimental test results. However the peak force in case of virtual test is $1.87 \mathrm{~N}$ and when compared to the experimental results the variation is $0.1 \mathrm{~N}$ (see Figure 11(a)-(b)). (ii) It is evident from the trajectory of the tripping force that only the front-side contacts are made. However the location of the contact points may vary from assembly to assembly. After careful study of the tripping force trajectory on the mass production samples, it is observed that the number of contacts may vary from sample to sample and the tripping force is also varying.

The results between the experimental and virtual model shows good match (see Figures 10(a)-(b) ??(a)-(b) and 11(a)-(b)). Once the virtual model is validated, it is ready for the virtual tests (see Figure ??). The virtual tests are carried in the similar way as the experimental test.

\section{Conclusions}

This paper is devoted to the numerical simulation of the C-60 circuit breaker built by Schneider Electric, using the so-called Moreau-Jean NSCD event-capturing numerical scheme. It relies on rigid body assumptions, with set-valued Coulomb's friction, and constant kinematic restitution coefficients. Emphasis is put on the modeling of three dimensional revolute joints with axial and radial clearance. Moreover detailed comparisons with experimental date obtained at the Schneider Electric laboratory, prove the very good prediction capabilities of the NSCD approach, for this type of mechanisms. 


\section{References}

1. ISO-1101: Geometrical Product Specifications (GPS) - Geometrical tolerancing - Tolerances of form, orientation, location and run-out. International Organization for Standardization (2012)

2. Abadie, M.: Dynamic simulation of rigid bodies: Modelling of frictional contact. In: B. Brogliato (ed.) Impacts in Mechanical Systems: Analysis and Modelling, Lecture Notes in Physics (LNP), vol. 551, pp. 61-144. Springer (2000)

3. Acary, V.: Projected event-capturing time-stepping schemes for nonsmooth mechanical systems with unilateral contact and Coulomb's friction. Computer Methods in Applied Mechanics and Engineering 256, 224-250 (2013)

4. Acary, V., Brogliato, B.: Numerical Methods for Nonsmooth Dynamical Systems. Applications in Mechanics and Electronics. Lecture Notes in Applied and Computational Mechanics 35. Berlin: Springer. xxi, 525 p. (2008)

5. Acary, V., Cadoux, F., Lemarechal, C., Malick, J.: A formulation of the linear discrete Coulomb friction problem via convex optimization. ZAMM-Journal of Applied Mathematics and Mechanics/Zeitschrift für Angewandte Mathematik und Mechanik 91(2), 155-175 (2011)

6. Akhadkar, N., Acary, V., Brogliato, B.: Analysis of collocated feedback controllers for four-bar planar mechanisms with joint clearances. Multibody System Dynamics 38(2), 101-136 (2016). DOI 10.1007/s11044-016-9523x. URL https://hal.inria.fr/hal-01218531

7. Brogliato, B.: Nonsmooth Mechanics: Models, Dynamics and Control, 2nd edn. Springer-Verlag, London (1999)

8. Colosimo, B., Senin, N.: Geometric Tolerances. Springer (2010)

9. De Saxcé, G.: Une généralisation de l'inégalité de Fenchel et ses applications aux lois constitutives. Comptes Rendus de l'Académie des Sciences t 314,série II, 125-129 (1992)

10. Flores, P.: A parametric study on the dynamic response of planar multibody systems with multiple clearance joints. Nonlinear Dynamics 61(4), 633-653 (2010)

11. Flores, P., R.Leine, Glocker, C.: Modeling and analysis of planar rigid multibody systems with translational clearance joints based on the non-smooth dynamics approach. Multibody System Dynamics 23, 165-190 (2010)

12. Gauthier, L.: General principles and rules for technical drawing. Technical Note FT15 057, Schneider Electric (2008)

13. Gummer, A., Sauer, B.: Influence of contact geometry on local friction energy and stiffness of revolute joints. Journal of tribology 134(2), 021,402 (2012)

14. Haroun, A., Megahed, S.: Simulation and experimentation of multibody mechanical systems with clearance revolute joints. In: Proceedings of World Academy of Science, Engineering and Technology, 63. World Academy of Science, Engineering and Technology (2012)

15. Jean, M.: The non-smooth contact dynamics method. Computer Methods in Applied Mechanics and Engineering 177(3), 235-257 (1999)

16. Jean, M., Moreau, J.: Dynamics in the presence of unilateral contacts and dry friction: a numerical approach. In: G. del Piero, F. Maceri (eds.) Unilateral Problems in Structural Analysis II, no. 304 in CISM Courses and Lectures, pp. 151-196. Springer Verlag (1987) 
17. Krinner, A., Thümmel, T.: Non-smooth behaviour of a linkage mechanism with revolute clearance joints. In: New Advances in Mechanisms, Transmissions and Applications, pp. 233-241. Springer (2014)

18. Orden, J.G.: Analysis of joint clearances in multibody systems. Multibody system dynamics 13(4), 401-420 (2005)

19. S.Yan, Xiang, W., Zhang, L.: A comprehensive model for $3 \mathrm{~d}$ revolute joints with clearances in mechanical systems. Nonlinear Dynamics pp. 1-20 (2014)

20. T. Thümmel and K. Funk: Multibody modelling of linkage mechanisms including friction, clearance and impact. In: Proceedings of the 10th World Congress on the Theory of Machines and Mechanisms in Oulu, June 20 to 24, vol. 4, pp. 1387-1392. Oulu University Press, Finland (1999) 